\title{
S17 Text. Post-epidemic demography
}

The 1400 epidemic inaugurated a prolonged fall in the number of heads of household registered as present in the marcs registers, from 1,997 in 1399 to 1,715 in 1400 , followed by a continuous decline to a nadir of 1,280 in 1422 . The $1338-1439$ epidemic resulted in a comparable fall from 1,780 in 1437 to 1,508 in 1439 , but was followed by a sustained increase, to 2,071 in the 1446 register, inaugurating the demographic recovery of the second half of the 15 th century $(3,070$ heads of household were present in 1501). 